\title{
State of Water in Hydrating Tricalcium Silicate and Portland Cement Pastes as Measured by Quasi-Elastic Neutron Scattering
}

\author{
Jeffrey J. Thomas ${ }^{\star}$ \\ Department of Civil Engineering, Northwestern University, Evanston, Illinois 60208 \\ Stephen A. FitzGerald \\ Department of Physics, Oberlin College, Oberlin, Ohio 44074
}

Dan A. Neumann

NIST Center for Neutron Research, National Institute of Standards and Technology, Gaithersburg, Maryland 20899

Richard A. Livingston*

Office of Infrastructure Research and Development, Federal Highway Administration, McLean, Virginia 22101

\begin{abstract}
Quasi-elastic neutron scattering (QENS) was used to monitor the state of water in portland cement and tricalcium silicate pastes during the first $\mathbf{2}$ days of hydration at three different temperatures. By applying a double-Lorentzian rather than a single-Lorentzian fitting function, the QENS signal from water at a given hydration time was divided into three separate populations arising from liquid water, chemically bound water, and constrained water. The constrained water population consisted of water adsorbed on surfaces and contained in very small $(<10 \mathrm{~nm})$ pores, and could be associated primarily with the calcium-silicate-hydrate $(\mathrm{C}-\mathrm{S}-\mathrm{H})$ phase. The rate of increase in the chemically bound water population closely followed the exothermic heat output, while the constrained water population increased more rapidly during the first several hours of hydration and then leveled off.
\end{abstract}

\section{Introduction}

$\mathrm{T}$ HE hydration of portland cement is a complex process involving several simultaneous chemical reactions. All of these reactions involve water, and as hydration proceeds, more of the water that is initially mixed with the cement powder becomes chemically bound into reaction product phases.

The most important reaction is between tricalcium silicate $\left(\mathrm{C}_{3} \mathrm{~S}^{\dagger}\right)$ and water, and pure $\mathrm{C}_{3} \mathrm{~S}$ powder is often used as a model system for cement. The hydration of $\mathrm{C}_{3} \mathrm{~S}$ can be written as

$$
\mathrm{C}_{3} \mathrm{~S}+(1.3+x) \mathrm{H} \Rightarrow \mathrm{C}_{1.7} \mathrm{SH}_{x}+1.3 \mathrm{CH}
$$

where $\mathrm{C}_{1.7} \mathrm{SH}_{x}$ is a loosely organized gel phase, known as C-S-H, that is the main binding component giving cement paste its strength, toughness, and durability.

During the first $24 \mathrm{~h}$ of hydration, C-S-H fills the space occupied by water in a $\mathrm{C}_{3} \mathrm{~S}$ or cement paste, forming a

M. Grutzeck—contributing editor

\footnotetext{
Manuscript No. 188815. Received January 13, 2000; approved November 2, 2000.

*Member, American Ceramic Society.
}

${ }^{\dagger}$ Cement chemistry notation: $\mathrm{C}=\mathrm{CaO}, \mathrm{S}=\mathrm{SiO}_{2}, \mathrm{H}=\mathrm{H}_{2} \mathrm{O}, \mathrm{A}=\mathrm{Al}_{2} \mathrm{O}_{3}, \overline{\mathrm{S}}=\mathrm{SO}_{3}$. high-surface-area product with an internal network of waterfilled gel pores. The C-S-H also contains chemically bound water and $\mathrm{OH}^{-}$groups, as well as adsorbed or "interlayer" water, which is less firmly bound. At later times, the C-S-H that forms has a monolithic morphology with no gel pores. The water content of $\mathrm{C}-\mathrm{S}-\mathrm{H}$ therefore varies considerably depending on the relative humidity of the paste, the age of the paste, and other processing variables. Under saturated conditions, when the gel pore water is included in the phase composition, the variable $x$ in Eq. (1) has a maximum value of about $4 .{ }^{1}$ The amount of chemically bound water associated with a welldefined solid C-S-H phase is considerably less, ranging from about $x=1.3$ to 2.1 depending on sample conditioning and the measurement technique used. ${ }^{1}$

Recently, quasi-elastic neutron scattering (QENS) has been used to follow the hydration of pure $\mathrm{C}_{3} \mathrm{~S}^{2,3}$ The QENS technique is sensitive to the state of hydrogen atoms in the paste, and the scattering signal can be separated into components arising from free (liquid) water and bound water. Because QENS measurements can be conducted in situ, without disturbing the reaction process, changes occurring during the early, rapid hydration stage can be measured.

Portland cement contains about $60 \% \mathrm{C}_{3} \mathrm{~S}$ by weight, and the hydration of $\mathrm{C}_{3} \mathrm{~S}$ is the most important reaction, particularly during the first few days of reaction. Other portland cement hydration reactions include the hydration of dicalcium silicate $\left(\mathrm{C}_{2} \mathrm{~S}\right)$ to form $\mathrm{C}-\mathrm{S}-\mathrm{H}$, which occurs much more slowly than reaction (1), and the hydration of tricalcium aluminate $\left(\mathrm{C}_{3} \mathrm{~A}\right)$ and gypsum $\left(\mathrm{CS}_{\mathrm{S}} \mathrm{H}_{2}\right)$ to form various aluminate phases such as monosulfate and ettringite. ${ }^{1}$ The aluminate phases do not contribute significantly to the strength development of cement paste except at very early times, but they do contain substantial amounts of water and would thus be expected to affect the QENS measurements.

The current study was performed using two different batches of $\mathrm{C}_{3} \mathrm{~S}$ powder and a commercial portland cement as the starting cementitious materials. For one set of experiments, isothermal hydration was followed for a period of approximately 2 days at one of three temperatures: $20^{\circ}, 30^{\circ}$, or $40^{\circ} \mathrm{C}$. For another series of experiments, performed on one of the $\mathrm{C}_{3} \mathrm{~S}$ powders, the hydration temperature was changed rapidly from one fixed value to another, to determine the sensitivity of the modeled free and bound water populations to temperature for a fixed 
microstructure. As a result of these experiments, the data analysis was modified to better account for the scattering response from water.

\section{Background}

The interactions of neutrons with atomic nuclei vary considerably with the element, with hydrogen providing by far the greatest cross section for scattering. As a consequence, the neutron scattering signal from a material containing a high proportion of elemental hydrogen is almost entirely from the hydrogen.

When a neutron collides with a chemically bound atom, it is scattered elastically; that is, its velocity and energy are unchanged. However, if the atom has rotational or translational freedom (for example, if it is diffusing through a liquid), the scattered neutron can gain or lose energy, depending on the relative movement of the atomic nucleus at the moment of the interaction. The interactions of neutrons with hydrogen atoms with various degrees of freedom are discussed by Livingston et $\mathrm{al}^{4}$

In a QENS experiment, a beam of monochromatic (fixed energy) neutrons is focused on the specimen, and the energy of the scattered neutrons is measured by determining the change in their velocity. The angle at which they are scattered is also measured. For a specimen with only bound atoms, a plot of scattering intensity versus final neutron energy will show a single sharp spike centered around the initial neutron energy, because all of the scattering will be elastic. The width of the spike is determined by the energy resolution of the instrument, and the intensity of such an elastic peak can be measured by fitting it with a Gaussian distribution.

The scattering response from diffusing atoms is quite different. Although the scattering response will still show an intensity peak centered on zero energy transfer, it will be broadened by the energy change imparted to the neutrons by the moving hydrogen nuclei. This type of peak is normally represented by a Lorentzian distribution. For liquid water, the width of the Lorentzian peak is a function of temperature and the scattering vector, with higher temperatures and larger scattering vectors giving a broader peak. In addition, the peak width of liquid water is affected by the presence of solid interfaces or small pores, which constrain the water molecules and thus cause narrowing of the peak. ${ }^{5}$ The degree of peak narrowing caused by this type of constraint depends both on the specific microstructure of the specimen and on the energy resolution of the instrument.

The QENS response from a hydrating cementitious paste can thus be represented by a superposition of a Gaussian peak and a Lorentzian peak. After the small Gaussian peak arising from the sample holder and the solid cement is subtracted, the relative intensity of the remaining peaks gives a measure of the fraction of hydrogen atoms in the bound and free states. The state of water in the sample can then be defined by a single parameter, such as the bound water index (BWI):

$$
\mathrm{BWI}=\frac{A}{A+B}
$$

where $A$ is the Gaussian intensity and $B$ is the Lorentzian intensity. By monitoring the change in the BWI with time, the kinetics of hydration can be determined.

\section{Experimental Procedure}

The isothermal hydration experiments were conducted using a type I portland cement (Lafarge Cement Co. ${ }^{*}$ ) and two different batches of triclinic $\mathrm{C}_{3} \mathrm{~S}$ powder. One batch (labeled $\mathrm{C}_{3} \mathrm{~S} \# 1$ ) was obtained from a commercial source (Construction Technology

${ }^{*}$ Manufacturers are identified in order to provide complete identification of experimental conditions, and such identification is not a recommendation or an endorsement by NIST.
Laboratories, Skokie, IL). This powder has an average particle size of $7.4 \mu \mathrm{m}$. The second batch (labeled $\mathrm{C}_{3} \mathrm{~S} \# 2$ ) was made by repeatedly calcining a $3: 1$ mixture of $\mathrm{CaO}$ and $\mathrm{SiO}_{2}$ powders at $1430^{\circ} \mathrm{C}$ and then grinding the resulting product in a mortar and pestle. ${ }^{\S} \mathrm{X}$-ray analysis indicated both powders to be pure triclinic $\mathrm{C}_{3} \mathrm{~S}$ with no unreacted $\mathrm{CaO}$. The QENS experiments involving a change in the hydration temperature were conducted using the $\mathrm{C}_{3} \mathrm{~S}$ \#1 powder.

In all experiments, the starting powder was combined with deionized water at a water-to-solids $(w / s)$ ratio of 0.4 by weight. The resulting paste was mixed by hand for $3 \mathrm{~min}$ and then spread into an aluminum sample holder, which was sealed with an indium gasket. A thin Teflon envelope prevented any direct contact between the aluminum holder and the paste. The internal dimensions of the sample holder were $\sim 6 \mathrm{~cm} \times 4 \mathrm{~cm} \times 1 \mathrm{~mm}$ thick. The thickness of the specimen was minimized to minimize multiple scattering effects, which greatly complicate data analysis. ${ }^{6}$ The temperature of the sample holder was carefully controlled and monitored throughout the run, and the neutron scattering response was measured continuously for $\sim 48 \mathrm{~h}$.

All of the QENS measurements were conducted using the Fermi chopper time-of-flight spectrometer at the NIST Center for Neutron Research in Gaithersburg, MD. ${ }^{7}$ The incident neutron wavelength was $4.8 \AA$, and the distance from the sample to the detectors was $2.29 \mathrm{~m}$, resulting in a calculated energy resolution of 0.146 $\mathrm{meV}$. To obtain sufficient statistics to accurately analyze the shape of the scattering function, the data from a range in scattering vector, $Q$, were summed together. The width of the energybroadened Lorentzian component of the scattering function increases with $Q$ at low values of $Q$, and then approaches a limiting value at high $Q$. Therefore, only high- $Q$ data were used. For each data point, the scattering was measured for $30 \mathrm{~min}$ and then all of the data from a $Q$ range of 1.9 to $2.4 \AA^{-1}$ were averaged together.

A differential scanning calorimeter (Hart Scientific, Pleasant Grove, UT) was used to monitor the isothermal heat evolution of pastes made from the same starting materials.

\section{Results}

\section{(1) Sensitivity of the Bound Water Index to Temperature}

To accurately compare the results of pastes hydrated at different temperatures, the separation of the overall signal intensity into different populations of hydrogen atoms must depend only on the microstructure of the paste and not on other variables, such as temperature. In other words, a given paste microstructure should have the same BWI at all temperatures. To test this, two $\mathrm{C}_{3} \mathrm{~S}$ pastes were hydrated with a sudden shift in the temperature at $24 \mathrm{~h}$. In one case the temperature was changed from $20^{\circ}$ to $40^{\circ} \mathrm{C}$, and in the other case it was changed from $40^{\circ}$ to $20^{\circ} \mathrm{C}$. These temperature changes occurred over a period of a few minutes, a time scale over which the microstructure of the paste can be assumed to remain fixed.

The data were initially analyzed by fitting the QENS response to Gaussian and Lorentzian components, each convoluted with the energy resolution of the instrument, as has been done in previous experiments. $^{2,3}$ This model for the scattering response can be written as ${ }^{3}$

$$
\begin{aligned}
S_{\text {inc }}(Q, \omega)= & C+\left\{A \delta(\omega=0)+B\left[\frac{\Gamma}{\pi\left(\Gamma^{2}+\omega^{2}\right)}\right]\right\} \\
& \otimes\left[\left(\frac{1}{\sigma \sqrt{2 \pi}}\right) \exp \left(-\omega^{2} / 2 \sigma^{2}\right)\right]
\end{aligned}
$$

${ }^{\S} \mathrm{C}_{3} \mathrm{~S}$ powder \#2, obtained courtesy of J. Francis Young, University of Illinois at Urbana-Champaign. 
where $S_{\text {inc }}$ is the incoherent scattering intensity, $Q$ is the scattering vector, $\omega$ is the energy change of a scattered neutron, $C$ is a $Q$-independent flat background, $\Gamma$ is the Lorentzian half-width at half-maximum, $\sigma$ is the energy resolution of the instrument, and $A$ and $B$ are the number density of bound and free hydrogen atoms, respectively. This is a four-parameter fit, with $A, B, C$, and $\Gamma$ varying to give the minimum $\chi^{2}$ between fit and data.

Figure 1 (open circles) shows the BWI for a paste hydrated at $20^{\circ} \mathrm{C}$ and then at $40^{\circ} \mathrm{C}$. At the temperature shift, the Gaussian component of the fit decreases and the Lorentzian component increases, causing the BWI of the specimen to decrease sharply. Similar behavior at the temperature shift was observed for the $40^{\circ}-20^{\circ} \mathrm{C}$ run, with the BWI also higher at $20^{\circ} \mathrm{C}$. This discontinuity is clearly caused by the fitting procedure rather than by a change in the microstructure. Moreover, as a paste hydrates at a fixed temperature, the width of the Lorentzian peak representing free water decreases significantly. ${ }^{3}$ These two results suggest that the single-Lorentzian function is modeling the combined response from more than one population of water. It is not surprising that under these circumstances a change in temperature, which changes the width of the Lorentzian peak, would appear to alter the BWI, contrary to the known invariance of the BWI with temperature as discussed above.

To resolve this discrepancy, the data from the temperature shift experiments were reanalyzed by fitting the experimental $S(Q, \omega)$ curves with two Lorentzian curves instead of one. The first Lorentzian was assigned a fixed width equal to the width of the free-water signal at the start of the reaction for a given temperature, and the width of the second Lorentzian was allowed to vary freely with time. The free-water widths were determined by measuring a sample holder filled with water at $20^{\circ}, 30^{\circ}$, and $40^{\circ} \mathrm{C}$ (see Table I). These independently determined values agreed closely with the widths of the single-Lorentzian fits at the start of a run, confirming that the water in a freshly mixed paste behaves like liquid water.

The above modifications add one degree of freedom to the original model (Eq. (3)). However, with the modified model the background was held fixed throughout the run, leaving the degrees of freedom unchanged at four. The modified model for the scattered intensity from hydrating paste can be written as

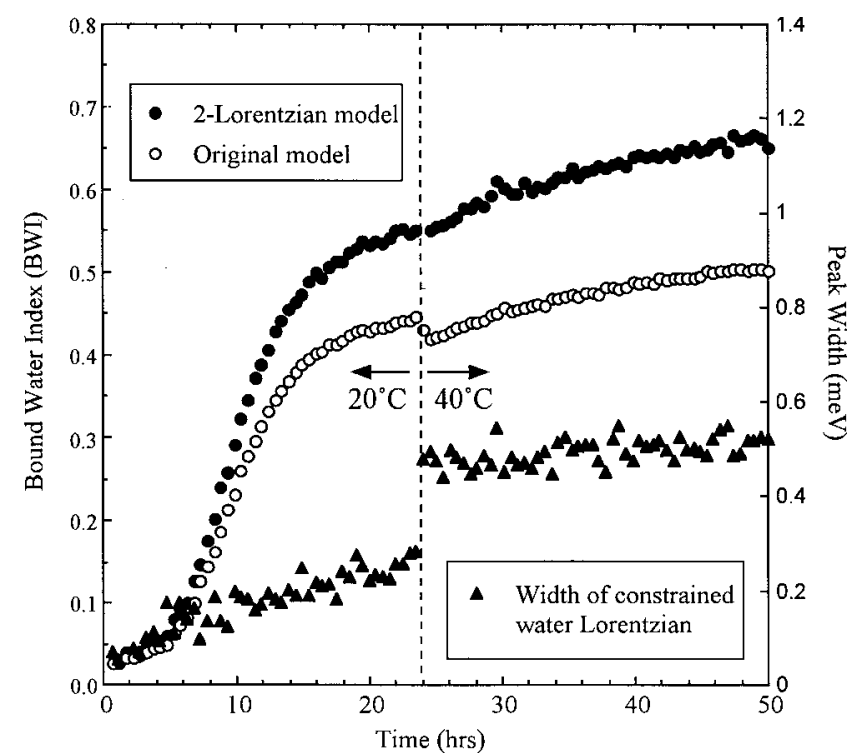

Fig. 1. Bound water index (BWI) for a $\mathrm{C}_{3} \mathrm{~S}$ paste hydrated at $20^{\circ} \mathrm{C}$ for $24 \mathrm{~h}$ and then at $40^{\circ} \mathrm{C}$ thereafter, calculated using the original singleLorentzian model (Eq. (3)) and the two-Lorentzian model defined by Eq. (4). The width of the second-Lorentzian peak $\left(\Gamma_{2}\right.$ in Eq. (4)) is also shown, using the right-hand axis.
Table I. Peak Widths of the Fitted Curves Associated with Different Populations of Water at Different Temperatures, and the Associated Activation Energies

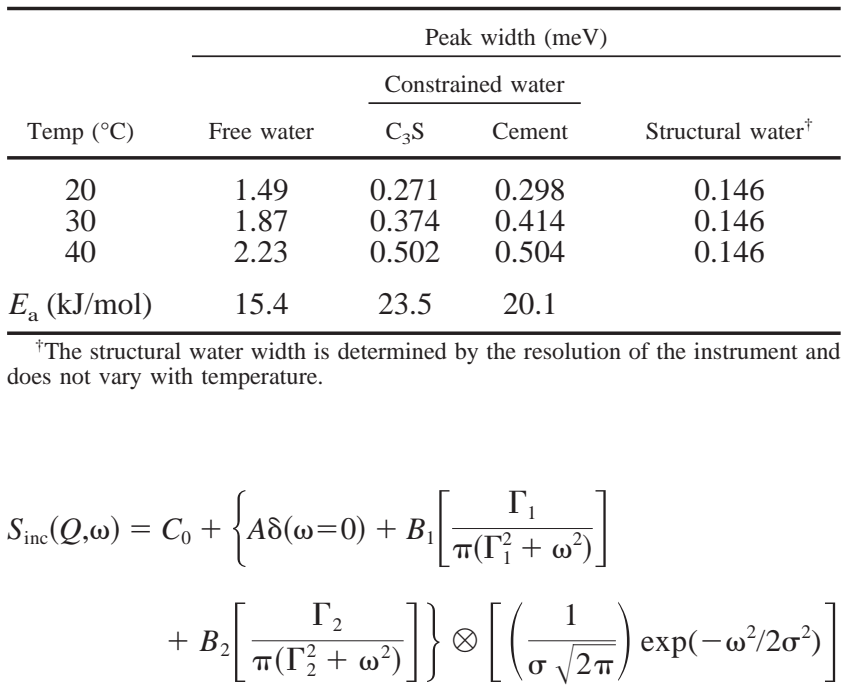

where $C_{0}$ is determined from the $S(Q, \omega)$ curve at the start of the run, $\Gamma_{0}$ is as given in Table I, and $A, B_{1}, B_{2}$, and $\Gamma_{2}$ are allowed to vary to minimize the $\chi^{2}$ of the fit.

The scattered intensity in the narrow Lorentzian peak is most likely associated with water in the nanoscale pore system of the $\mathrm{C}-\mathrm{S}-\mathrm{H}$, and thus arises from reaction product. We therefore include the intensity from this peak in the total bound water population, redefining the BWI as

$$
\mathrm{BWI}=\frac{A+B_{2}}{A+B_{1}+B_{2}}
$$

With this double-Lorentzian model, the scattered intensity in the Gaussian peak (parameter $A$ ) is associated only with chemically bound hydrogen atoms. For purposes of clarity this population will be referred to as "structural" water, while the population associated with the narrow Lorentzian (parameter $B_{2}$ ) will be referred to as "constrained" water. The sum of the structural and constrained water peaks $\left(A+B_{2}\right)$ gives the total bound water in the hydrating paste. As with the previous model, the liquid (free) water is represented by the fixed-width Lorentzian peak (parameter $B_{1}$ ).

Figure 1 (closed circles) shows the BWI obtained from the modified model defined by Eqs. (4) and (5) for the $\mathrm{C}_{3} \mathrm{~S}$ paste hydrated with a temperature shift from $20^{\circ}$ to $40^{\circ} \mathrm{C}$. The sharp discontinuity at the temperature shift has been eliminated, indicating that the double-Lorentzian model distinguishes between free and bound water on a more physically realistic basis. The reanalyzed data for the $\mathrm{C}_{3} \mathrm{~S}$ paste hydrated with a temperature shift from $40^{\circ}$ to $20^{\circ} \mathrm{C}$ also resulted in a BWI that was smooth across the temperature shift.

The width of the narrow, variable-width Lorentzian, $\Gamma_{2}$, is an important variable to consider when assessing the physical basis for the modified model, because it is allowed to vary freely during the fit. Figure 1 also shows $\Gamma_{2}$ plotted against hydration time for the two $\mathrm{C}_{3} \mathrm{~S}$ hydration runs conducted with a temperature shift (closed triangles, right axis). After some initial variation when the intensity is very low, the width reaches a constant value, and then changes sharply to a new value at the temperature shift. The width is larger at $40^{\circ} \mathrm{C}$, due to the increased rate of diffusional motion of the water molecules with temperature. ${ }^{8}$

These results confirm that in addition to the liquid and structural water populations, there is a third population of water in the paste which is constrained, or loosely bound, so that it scatters neutrons with a narrower Lorentzian energy width which is close to the instrument energy resolution. With the earlier single-Lorentzian model (Eq. (3)), the intensity from this population was divided between the Gaussian and Lorentzian peaks. As the temperature 
increased, the constrained water peak broadened (while the Gaussian width remained fixed), shifting intensity out of the modeled Gaussian peak and into the modeled Lorentzian peak. As a consequence, the calculated BWI decreased with increasing temperature.

\section{(2) Effect of Temperature on the Isothermal Hydration of $\mathrm{C}_{3} \mathrm{~S}$ and Cement Paste}

The calculation of a physically sound BWI using the doubleLorentzian method makes it possible to make valid comparisons among samples of pastes that have been cured under different conditions. In this case, the $\mathrm{C}_{3} \mathrm{~S}$ and cement pastes were hydrated isothermally at $20^{\circ}, 30^{\circ}$, and $40^{\circ} \mathrm{C}$ for $\sim 2$ days. Based on the results of the previous section, the individual energy spectra were modeled by fitting them to three convoluted curves, as given by Eq. (4). This resulted in constrained water populations with Lorentzian widths that increased with temperature but were similar for the two types of pastes (see Table I). This peak broadening can be fitted with an Arrhenius plot to extract apparent activation energies (see Fig. 2 and Table I). The constrained water populations have a higher activation energy than the free water population.

Figure 3 shows the BWI of the two $\mathrm{C}_{3} \mathrm{~S}$ pastes and the cement paste for isothermal hydration at $30^{\circ} \mathrm{C}$. For all three pastes, the BWI increases rapidly for the first several hours and then levels out, which is the standard kinetic profile for the hydration of cement and $\mathrm{C}_{3} \mathrm{~S} .{ }^{1}$ After showing significantly different rates initially, all three pastes converge to the same curve between 2 and $11 \mathrm{~h}$. This period agrees very well with the phase of peak nucleation and growth identified by FitzGerald et $a l^{3}$ for $\mathrm{C}_{3} \mathrm{~S}$ hydrating isothermally at $30^{\circ} \mathrm{C}$. The fact that samples of three different compositions show similar kinetics implies that the rate-controlling process during this phase is independent of cement composition or surface and hence involves a through-solution mechanism. It also suggests that it might be possible to develop a universal hydration curve for a wide range of cement compositions for this part of the reaction using $\mathrm{C}_{3} \mathrm{~S}$ as a model system.

However, there are significant differences between the cement and $\mathrm{C}_{3} \mathrm{~S}$ pastes before and after the peak rate period (see Fig. 3 ). First, the BWI at the start of the reaction is higher for cement paste than for $\mathrm{C}_{3} \mathrm{~S}$ paste. We hypothesize that this is due to the rapid early formation of hydrated aluminate sulfate phases in the portland cement system. Within the first 10 min after mixing, cement particles become coated with a layer of ettringite, an

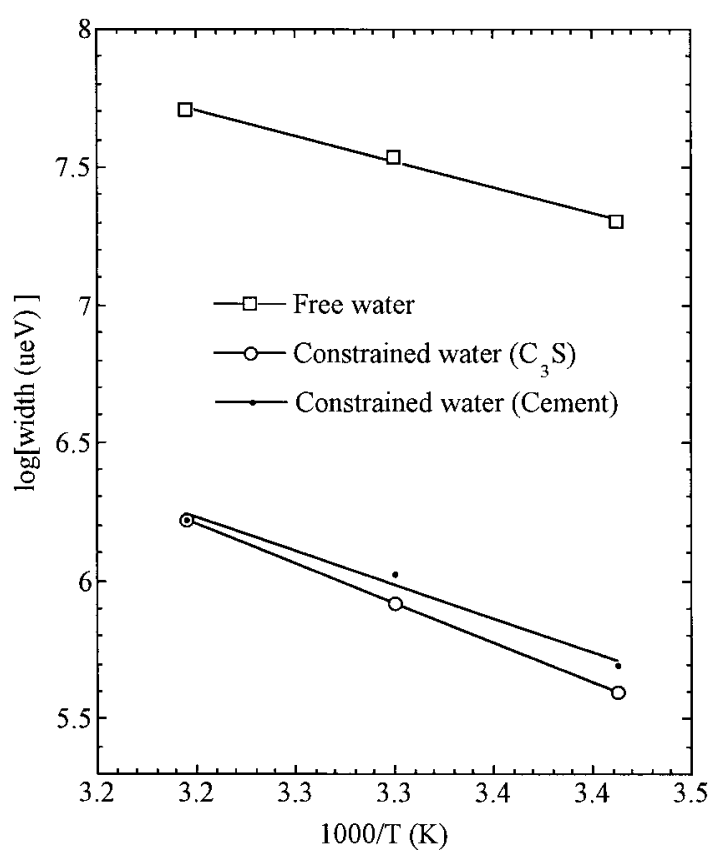

Fig. 2. Arrhenius plot of the Lorentzian peak widths. The activation energies are given in Table I.

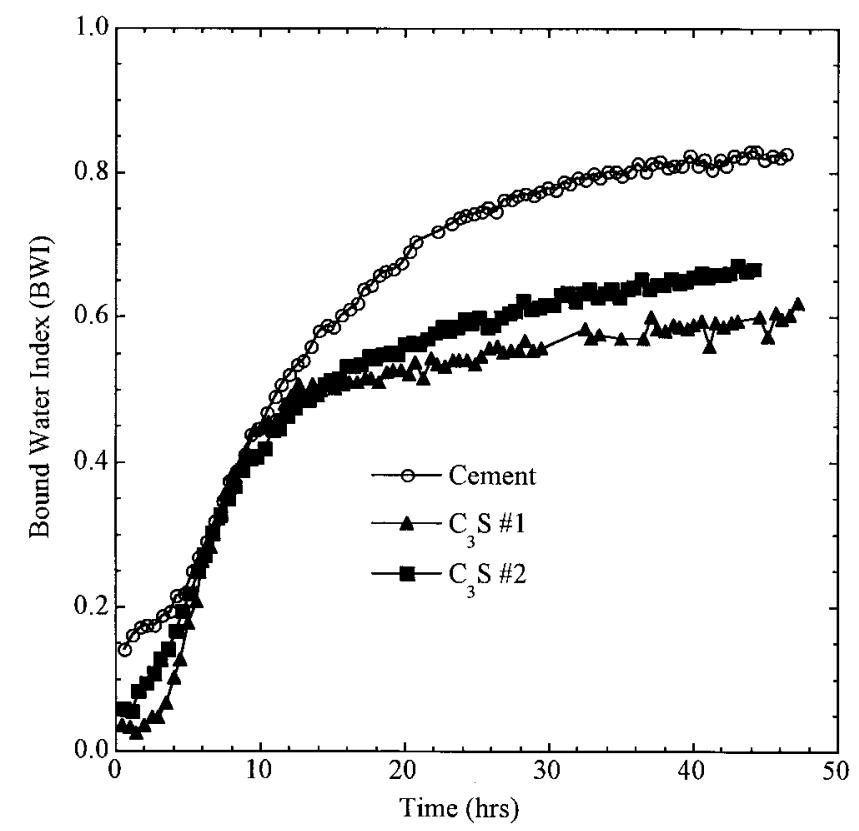

Fig. 3. BWI as calculated using Eqs. (4) and (5) as a function of time for isothermal hydration at $30^{\circ} \mathrm{C}$.

aluminate-sulfate phase high in water. ${ }^{1}$ This phase would thus be present by the time the first measurements were made, accounting for the higher initial BWI.

There are also differences in the shapes of the curves at the end of the peak rate period as the hydration reaction becomes diffusion-limited. In the $\mathrm{C}_{3} \mathrm{~S}$ pastes, the transition to the slow growth rate is sharp. This behavior also exists in the cement system, but the curves are not as steep and the transition to diffusion-limited behavior is more gradual. This is what would be expected when comparing a pure system $\left(\mathrm{C}_{3} \mathrm{~S}\right)$ to a system with several components reacting at different rates (cement). In a portland cement paste, the reaction of $\mathrm{C}_{2} \mathrm{~S}$ to form $\mathrm{C}-\mathrm{S}-\mathrm{H}$ and the conversion of high-water aluminate phases such as ettringite to low-water phases such as monosulfate occur in parallel with the $\mathrm{C}_{3} \mathrm{~S}$ hydration reaction. Morphologic differences in the $\mathrm{C}-\mathrm{S}-\mathrm{H}$ formed in cement and $\mathrm{C}_{3} \mathrm{~S}$ pastes may affect the diffusion characteristics as well.

Finally, the cement pastes reach a higher BWI after 2 days of hydration than do the $\mathrm{C}_{3} \mathrm{~S}$ pastes. This is again primarily due to the formation of aluminate phases such as ettringite and monosulfate in the cement pastes, which bind up considerable amounts of water. All of the trends illustrated by Fig. 3 at $30^{\circ} \mathrm{C}$ hold at the other temperatures as well.

The BWIs at all three temperatures are shown in Fig. 4 for all three pastes, allowing the effect of hydration temperature on a given paste to be observed. For each paste, the initial rate of increase of the BWI increases with temperature, in agreement with previous QENS results and with isothermal calorimetry. For the $\mathrm{C}_{3} \mathrm{~S}$ pastes, the BWI reached after 1-2 days of hydration is not always higher at higher temperatures. In particular, the $40^{\circ} \mathrm{C}$ runs reach slightly lower BWI values than the other runs. This type of "crossover" behavior has been reported for the degree of hydration versus time of $\mathrm{C}_{3} \mathrm{~S}$ pastes, ${ }^{9,10}$ although for a greater range of hydration temperature. Such an inverse temperature dependence could be accounted for by differences in the morphology of the early C-S-H product layer, with higher temperatures producing a less permeable product which inhibits the diffusion-controlled growth of C-S-H at later times. ${ }^{9}$ Analysis of the QENS data for the rate of increase of the BWI in the diffusion-controlled regime also supports this hypothesis; this is the subject of a separate paper. ${ }^{11}$

It should be noted that the relationship between the BWI of a paste and the degree of hydration (defined as the fraction of $\mathrm{C}_{3} \mathrm{~S}$ consumed) depends on the H/S molar ratio of the C-S-H (variable $x$ in Eq. (1), and this does not necessarily remain constant with 

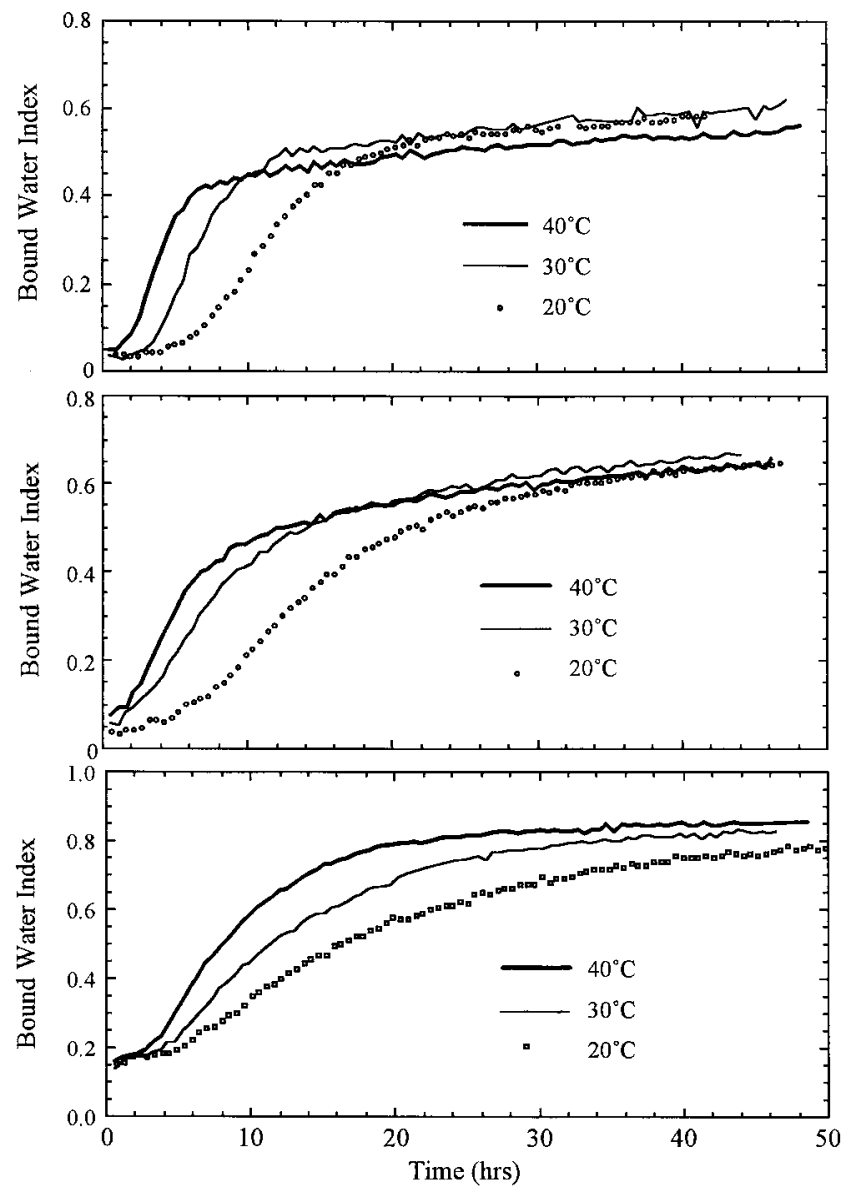

Fig. 4. BWI as a function of time for isothermal hydration at $20^{\circ}, 30^{\circ}$, and $40^{\circ} \mathrm{C}$ : (top) $\mathrm{C}_{3} \mathrm{~S} \# 1$, (middle) $\mathrm{C}_{3} \mathrm{~S} \# 2$, (bottom) cement. Note different vertical scales.

temperature. For example, Bentur et al. ${ }^{10}$ found that a $\mathrm{C}_{3} \mathrm{~S}$ paste hydrated at $65^{\circ} \mathrm{C}$ had a lower $\mathrm{H} / \mathrm{S}$ ratio than pastes hydrated at $4^{\circ}$ or $25^{\circ} \mathrm{C}$.

The results for $\mathrm{C}_{3} \mathrm{~S}$ paste \#1 shown in Fig. 4 do not agree with previously published QENS data for the hydration of this batch of $\mathrm{C}_{3} \mathrm{~S}$ at different temperatures, ${ }^{3}$ which found that the BWI reached after 2 days of hydration had a much stronger inverse temperature dependence. The earlier results were obtained using the singleLorentzian model described by Eq. (3) to fit the free-water portion of the energy spectrum. As discussed in Section IV(1), this caused a higher proportion of the total scattered intensity to be assigned to bound water at lower temperatures for a given paste microstructure.

\section{(3) Structural and Constrained Water Content of C-S-H}

Table II lists the fraction of structural, constrained, and free

Table II. Percentages of Structural, Constrained, and Free Water in the Pastes after Two Days of Hydration

\begin{tabular}{lcccc}
\hline Paste & $T\left({ }^{\circ} \mathrm{C}\right)$ & Structural $(\%)$ & Constrained $(\%)$ & Free \\
\hline $\mathrm{C}_{3} \mathrm{~S} \# 1$ & 20 & 43.7 & 17.5 & 38.8 \\
$\mathrm{C}_{3} \mathrm{~S} \# 1$ & 30 & 44.1 & 17.7 & 38.2 \\
$\mathrm{C}_{3} \mathrm{~S} \# 1$ & 40 & 41.6 & 16.7 & 41.7 \\
$\mathrm{C}_{3} \mathrm{~S} \# 2$ & 20 & 46.2 & 20.6 & 33.2 \\
$\mathrm{C}_{3} \mathrm{~S} \# 2$ & 30 & 45.6 & 21.4 & 33.0 \\
$\mathrm{C}_{3} \mathrm{~S} \# 2$ & 40 & 42.9 & 22.6 & 34.6 \\
OPC & 20 & 60.1 & 17.4 & 22.5 \\
OPC & 30 & 63.8 & 18.8 & 17.4 \\
OPC & 40 & 65.7 & 19.7 & 14.6 \\
\hline
\end{tabular}

water in each paste after 2 days of hydration. For a given paste, the fractional values are relatively independent of the hydration temperature, but there are considerable differences between $\mathrm{C}_{3} \mathrm{~S}$ and OPC pastes. OPC pastes have a higher percentage of structural water and lower amounts of constrained and free water. This can be attributed to the formation of aluminate phases such as ettringite in OPC paste. The lower percentage of constrained water in OPC pastes suggests that despite the higher BWI (see Figs. 3 and 4) the amount of C-S-H formed after 2 days is lower. This is sensible, given that $\mathrm{C}-\mathrm{S}-\mathrm{H}$ formation at early times comes almost entirely from $\mathrm{C}_{3} \mathrm{~S}$, which makes up about $60 \mathrm{wt} \%$ of the OPC. It should be noted that the C-S-H formed from pure $\mathrm{C}_{3} \mathrm{~S}$ and from OPC do not necessarily have identical morphology, composition, or structure. It can be assumed that these differences are negligible, however. ${ }^{1}$

The structural and constrained water populations have different kinetics during the first 2 days of hydration. The constrained water intensity increases very rapidly during the first several hours of hydration and then levels off almost completely, showing little increase after about $24 \mathrm{~h}$ of reaction. The structural water intensity also increases rapidly during the first several hours, but does not level off completely. Figure 5 shows the normalized kinetics for the structural water intensity, the constrained water intensity, and the total heat evolved as measured by thermal calorimetry for the OPC paste hydrated at $30^{\circ} \mathrm{C}$. For each of these normalized variables, zero represents the value at the end of the induction period ( $\sim 1 \mathrm{~h}$ after mixing) and 1 represents the value at $48 \mathrm{~h}$. The structural water intensity follows the heat evolution very closely, while the constrained water intensity has a clearly different shape.

The kinetics of the constrained water intensity have a parallel in the development of surface area in cement pastes as measured by small-angle neutron scattering. The specific surface area of a hydrating paste was found to increase very rapidly for the first $18 \mathrm{~h}$ and then to level off completely, showing little or no increase over the next 28 days despite continued hydration as measured by the evolution of heat. ${ }^{12}$ This was interpreted as evidence for two distinct morphologies of the C-S-H: a high surface area form which rapidly fills the capillary porosity at the start of the hydration process, and a denser form which forms at a slower rate and which has essentially no surface area. These morphologies are likely related to the "outer product" and "inner product" morphologies of C-S-H which are observed by microscopy (see Ref. 1, p. 123).

The current results agree with this hypothesis, in that the constrained water population can be associated solely with the high-surface-area form of C-S-H. In contrast, both outer and inner product C-S-H would contain chemically bound (structural) water. The close correlation between the structural water intensity measured by QENS and the heat evolution (see Fig. 5) supports the

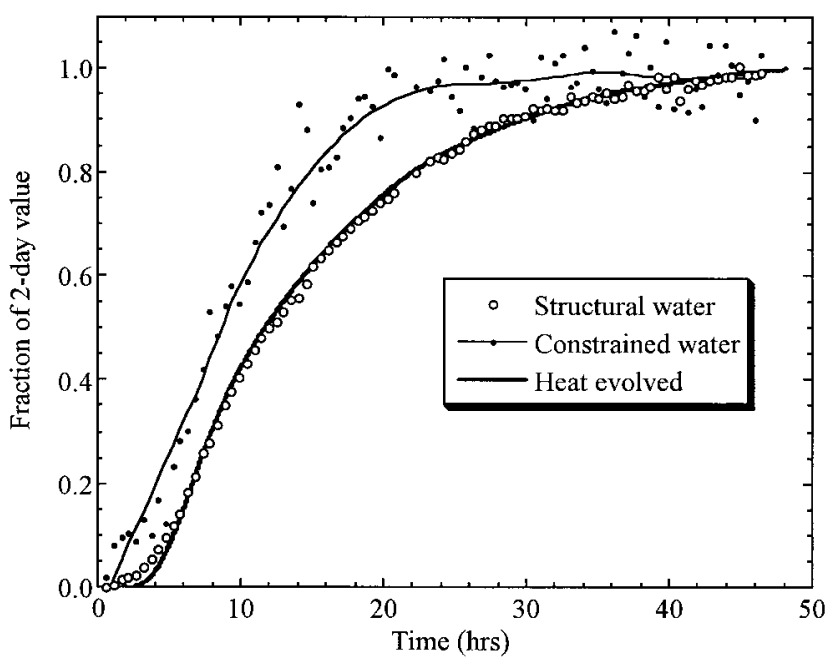

Fig. 5. Normalized plots of structural water, constrained water, and heat evolved for cement paste hydrated at $30^{\circ} \mathrm{C}$. The line through the constrained water points is a smooth (moving average) fit to the data. 
logical assumption that the chemical binding of water and the release of heat are directly related.

On a molar basis, the starting $w / s$ ratio of 0.4 for the $\mathrm{C}_{3} \mathrm{~S}$ pastes gives $5.07 \mathrm{~mol}$ of water for each mole of $\mathrm{C}_{3} \mathrm{~S}$. Using the $\mathrm{C}_{3} \mathrm{~S} \# 2$ paste hydrated at $30^{\circ} \mathrm{C}$ as an example, the data in Table II imply that after 2 days of hydration there are $2.31 \mathrm{~mol}$ of structural water, $1.09 \mathrm{~mol}$ of constrained water, and $1.67 \mathrm{~mol}$ of free water for each mole of original $\mathrm{C}_{3} \mathrm{~S}$ powder. In a $\mathrm{C}_{3} \mathrm{~S}$ paste, the bound and constrained hydrogen atoms can be associated with only two phases: C-S-H and calcium hydroxide. Calcium hydroxide is a crystalline phase and thus contains only structural water, while C-S-H contains some structural water and all of the constrained water.

Assuming that the hydration of $\mathrm{C}_{3} \mathrm{~S}$ proceeds according to Eq. (1), the amount of calcium hydroxide that has formed from $1 \mathrm{~mol}$ of $\mathrm{C}_{3} \mathrm{~S}$ at a given degree of hydration $(\alpha)$ is $1.3 \alpha$. The total heat evolved for this paste after 2 days of hydration at $30^{\circ} \mathrm{C}$ was determined to be $62.3 \mathrm{~kJ} / \mathrm{mol}$. Assuming the enthalpy of hydration of $\mathrm{C}_{3} \mathrm{~S}$ to be $120 \mathrm{~kJ} / \mathrm{mol},{ }^{1}$ this gives a degree of hydration of $\alpha=$ 0.52 , and this implies that $0.7 \mathrm{~mol}$ of $\mathrm{CH}$ has formed. The remainder of the $2.3 \mathrm{~mol}$ of structural water must be associated with C-S-H, resulting in a formula of $\mathrm{C}_{1.7} \mathrm{SH}_{\mathrm{S}(1.6)}$, where $\mathrm{H}_{\mathrm{S}}$ is structural water measured by QENS. Including the constrained water population in the C-S-H results in the formula $\mathrm{C}_{1.7} \mathrm{SH}_{\mathrm{T}(2.7)}$, where $\mathrm{H}_{\mathrm{T}}$ is the total bound water measured by QENS. Given the relative kinetics of formation of the constrained and structural water populations shown in Fig. 5, the $\mathrm{H}_{\mathrm{T}} / \mathrm{S}$ molar ratio of the $\mathrm{C}-\mathrm{S}-\mathrm{H}$ would be highest during the early acceleratory period of hydration and would decrease gradually thereafter as new $\mathrm{C}-\mathrm{S}-\mathrm{H}$ with a lower H/S ratio continues to form.

The estimated H/S molar ratios of 1.6 and 2.7 can be compared with values of $\mathrm{H} / \mathrm{S}$ for $\mathrm{C}-\mathrm{S}-\mathrm{H}$ in $\mathrm{C}_{3} \mathrm{~S}$ pastes measured directly using other techniques (see Ref. 1). The C-S-H in saturated $\mathrm{C}_{3} \mathrm{~S}$ paste has an $\mathrm{H} / \mathrm{S}$ value of about 4 , which includes free water contained within the gel pores. When the paste is equilibrated to $11 \%$ relative humidity, a procedure designed to remove water from the gel pores but not from interlayer spacings, $\mathrm{H} / \mathrm{S}$ decreases to about 2.1. Drying $\mathrm{C}_{3} \mathrm{~S}$ paste under vacuum or in a $105^{\circ} \mathrm{C}$ oven, procedures which remove all loosely adsorbed and pore water as well as some of the interlayer water, results in measured $\mathrm{H} / \mathrm{S}$ values ranging from 1.3 to 1.5 . The value $\mathrm{H}_{\mathrm{S}} / \mathrm{S}=1.6$ measured by QENS is slightly higher than the measured water content of C-S-H after strong drying, suggesting that the structural water measured by QENS includes both interlayer and chemically bound water. The total bound water content, $\mathrm{H}_{\mathrm{T}} / \mathrm{S}=2.7$, is intermediate between the values that include all and none of the gel pore water. This analysis supports the assumption that the constrained water population measured by QENS is associated with water contained in the smallest C-S-H pores and adsorbed on solid-pore interfaces.

\section{Conclusions}

Quasi-elastic neutron scattering allows the hydrogen atoms in the mix water of a cementitious paste to be monitored as the hydration reaction proceeds and the water is bound into product phases. The application of a double-Lorentzian fit instead of the single Lorentzian used in previous work leads to a refinement of the distribution of the water among the various states. Three distinct populations corresponding to free (liquid) water, structural water, and constrained water have been identified, and their relative intensities were measured for tricalcium silicate and portland cement pastes during the first 2 days of hydration at three different temperatures. The constrained water population can be associated with water adsorbed onto (and contained within) the nanoscale gel pores of the $\mathrm{C}-\mathrm{S}-\mathrm{H}$ reaction product.

The increase in the total bound water population (structural plus constrained water) with time gives a measure of the hydration kinetics. During the period of initial fast hydration, the rate of hydration is strongly temperature-dependent, in agreement with measurements of the rate of heat output. The convergence of the hydration curves during this phase implies that $\mathrm{C}_{3} \mathrm{~S}$ provides a reliable model system for this period. For $\mathrm{C}_{3} \mathrm{~S}$ pastes, the transition to slower diffusion-controlled kinetics resulted in some "crossover," whereby the pastes hydrated at $40^{\circ} \mathrm{C}$ had less bound water after 2 days than pastes hydrated at $20^{\circ} \mathrm{C}$. This can be associated in large part to changes with temperature in the permeability of the early product layer that surrounds the $\mathrm{C}_{3} \mathrm{~S}$ particles.

The increase of the structural water population tracks with the total heat output of the pastes very closely during the first 2 days of hydration, while the increase in the constrained water population has a different profile. These results support the hypothesis that two distinct morphologies of the C-S-H form in hydrating pastes: a high-surface-area form containing water-filled gel pores that rapidly fills the capillary pore space, and a monolithic low-surface-area form that surrounds and replaces the original $\mathrm{C}_{3} \mathrm{~S}$ particles.

\section{References}

${ }^{1}$ H. F. W. Taylor, Cement Chemistry, 2nd ed. Telford, London, U.K., 1997.

${ }^{2}$ R. Berliner, M. Popvici, K. W. Herwig, M. Berliner, H. M. Jennings, and J. J. Thomas, "Quasi-Elastic Neutron Scattering Study of the Effect of Water to Cement Ratio on the Hydration Kinetics of Tricalcium Silicate," Cem. Concr. Res., 28 [2] 231-44 (1998).

${ }^{3}$ S. A. FitzGerald, D. A. Neumann, J. J. Rush, D. P. Bentz, and R. A. Livingston, "In Situ Quasi-Elastic Neutron Scattering Study of the Hydration of Tricalcium Silicate," Chem. Mater., 10 [1] 397-402 (1998).

${ }^{4}$ R. A. Livingston, D. A. Neumann, A. J. Allen, and J. J. Rush, "Application of Neutron Scattering Methods to Cementitious Materials," Mater. Res. Soc. Symp. Proc., 376, 459-69 (1995).

${ }^{5}$ J. M. Zanotti, M. C. Bellissent-Funel, and S. H. Chen, "Relaxational Dynamics of Supercooled Water in Porous Glass," Phys. Rev. E, 59, 3084-93 (1999).

${ }^{6}$ M. Bée, Quasielastic Neutron Scattering; pp. 107-47. Hilger, Bristol, U.K., 1988.

7 J. R. D. Copley and T. J. Udovic, "Neutron Time-of-Flight Spectroscopy," J. Res. Natl. Inst. Stand. Technol., 98, 71 (1993).

${ }^{8}$ J. Teixera, M.-C. Bellisent-Funel, S. H. Chen, and A. J. Dianoux, "Experimental Determination of the Nature of Diffusive Motions of Water Molecules at Low Temperatures," Phys. Rev. A, 31, 1913 (1985).

${ }^{9}$ S. Brunauer and D. L. Kantro; pp. 287-309 in The Chemistry of Cements, Vol. 1. Edited by H. F. W. Taylor. Academic Press, New York, 1964.

${ }^{10}$ A. Bentur, R. L. Berger, J. H. Kung, N. B. Milestone, and J. F. Young, "Structural Properties of Calcium Silicate Pastes: II, Effect of Curing Temperature," J. Am. Ceram. Soc., 62, 362 (1979).

${ }^{11}$ S. A. FitzGerald, J. J. Thomas, D. A. Neumann, and R. A. Livingston, "A Neutron Scattering Study of the Role of Diffusion in the Hydration of Tricalcium Silicate," Cem. Concr. Res., submitted.

${ }^{12}$ J. J. Thomas, H. M. Jennings, and A. J. Allen, "The Surface Area of Cement Paste as Measured by Neutron Scattering_Evidence for Two C-S-H Morphologies," Cem. Concr. Res., 28 [6] 897-905 (1998). 